\title{
Consistency Properties of a Chaotic Semiconductor Laser Driven by Optical Feedback
}

\author{
Neus Oliver, Thomas Jüngling," and Ingo Fischer \\ Instituto de Física Interdisciplinar y Sistemas Complejos, IFISC (UIB-CSIC), Campus Universitat de les Illes Balears, \\ E-07122 Palma de Mallorca, Spain
}

(Received 12 November 2014; published 26 March 2015)

\begin{abstract}
We experimentally study consistency properties of a semiconductor laser in response to a coherent optical drive originating from delayed feedback. The laser is connected to a short and a long optical fiber loop, switched such that only one is providing input to the laser at a time. This way, repeating the exact same optical drive twice, we find consistent or inconsistent responses depending on the pump parameter and we relate the kind of response to strong and weak chaos. Moreover, we are able to experimentally determine the sub-Lyapunov exponent, underlying the consistency properties.
\end{abstract}

DOI: 10.1103/PhysRevLett.114.123902

PACS numbers: 42.65.Sf, 05.45.Tp, 05.45.Xt, 42.55.Px

Consistency is a general concept describing the reproducibility of responses of a dynamical system when repeatedly driven by similar inputs, each time, however, starting from different initial conditions [1]. Thus, it characterizes the property of whether a similar drive results in a similar response. Although the definition of similarity lacks precision, consistency is a powerful concept with implications in various fields. Consistency in the dynamics of neurons is a necessary condition for the reliability in cognitive tasks [2]. Moreover, it is a key ingredient to hardware- and software-based neuroinspired information processing techniques like reservoir computing [3-8]. Therefore, it is crucial to gain a thorough understanding of this property and to achieve a high level of control over it. A strong form of consistency, when the dynamical system converges towards the same response to an input, is closely related to generalized synchronization [9-12]. While consistency has been introduced in a context where the same system is driven repeatedly [1], in the case of generalized synchronization one considers two replicas of the same system that are subject to the same drive [13]. The underlying mechanism of both phenomena is the same and relies on the conditional stability of the driven system [14]. Recent experimental and numerical advances comprise generalized synchronization properties of laser systems driven by a common light source with fluctuating phase and/or amplitude [15-19].

In this Letter, we address two challenging aspects of consistency. The first is the development of an experimental scheme providing a high quality repeated drive for fast experimental systems with nonscalar drive signals. The second is the application, extension, and interpretation of time series analysis methods in order to reveal insights into the mechanisms underlying consistency. A remarkable phenomenon in this context is that the same dynamical system might show a consistent response to a certain complex drive and an inconsistent response to another. Such transitions can occur by variation of a single parameter [17,20,21]. Here, we concentrate on consistency of a semiconductor laser subject to a drive originating from time-delayed optical feedback. The property that delayed optical feedback induces high dimensional chaotic dynamics in semiconductor lasers has been studied in detail over the last decades [22]. Here, we use this mechanism as an ideal generator for complex optical drive signals.

In the framework of semiconductor laser dynamics, the consistency-related aspects of strong and weak chaos in delay systems were recently introduced [23]. A fully consistent response of the laser to its own chaotic delayed signal is referred to as weak chaos, whereas an inconsistent response corresponds to strong chaos. Only by changing the feedback or pump parameters are there nontrivial transitions between these different states, which were identified in numerical simulations of a semiconductor laser rate equation model [24]. Experimentally, there are only indirect indications for these transitions like spectral or intensity autocorrelation signatures of the laser dynamics in the case of delayed feedback [25] or of delay-coupled lasers [26]. A direct identification requires the knowledge of the sub-Lyapunov exponent [24]. It characterizes the stability of the response of a dynamical system to its delayed feedback analog to the conditional Lyapunov exponent of an externally driven system [14,20,21,27]. In particular, a negative sub-LE describes the convergence of the response trajectories to a function of the feedback drive, whereas a positive sub-LE describes their divergence. Although the sub-LE is at the core of the consistency property, so far it has not been possible to determine it experimentally.

We adapted the idea of a repeated drive [1] to the case of coherent and high bandwidth optical drive signals. We particularly consider a semiconductor laser exhibiting chaotic dynamics due to a delayed feedback drive. Our scheme is based on two switchable feedback loops with significantly different lengths. This allows us to store the optical drive with all its features in the long fiber loop while 
the primary short loop is active and to replay it while the short loop is blocked. The replay offers high accuracy without practical limitations with respect to optical and dynamical bandwidth. Even the optical phase dynamics is maintained. Moreover, in comparison to an equivalent synchronization scheme using a twin system, we minimize mismatches by construction, because the identical laser is driven by the repeated signals. This, in combination with the high quality of the replay, even allows for access to a measure proportional to the sub-LE, directly quantifying the consistency properties.

Figure 1 shows the simplified experimental scheme. The laser diode is an Eblana Discrete Mode Laser lasing at $1.5 \mu \mathrm{m}$ with a threshold current of the solitary laser of $I_{\mathrm{th}}=11.8 \mathrm{~mA}$. The laser light is split to simultaneously enter two optical fiber loops, a short one with a roundtrip time of $\tau_{1}=111 \mathrm{~ns}$ and a much longer one with $\tau_{2}=21 \mu \mathrm{s}$. At the end of each fiber loop the light passes through a Mach-Zehnder modulator. These electro-optical modulators are controlled by a pulse generator with a $50 \%$ duty cycle, such that when one path is open, the other one is blocked. The period of the modulation is set to $50 \mu \mathrm{s}$ and the switching time of the pulses is $<200$ ps. During the first $25 \mu \mathrm{s}$, the laser receives feedback from the short delay loop, giving rise to the characteristic chaotic delay dynamics. When the short path is closed and the long delay loop is opened, the laser receives a replay of the optical field it already received a time $\tau_{2}-\tau_{1}$ before. We carefully designed and adjusted the system to obtain an exact repetition of the previous optical field. The levels of the two drive signals are adjusted to a precision of $\pm 0.01 \mathrm{~dB}$ and the suppression of the blocked loop is $>20 \mathrm{~dB}$ [12]. The laser output is detected with a photodiode of $12.5 \mathrm{GHz}$ bandwidth and a digital oscilloscope with $16 \mathrm{GHz}$ analog bandwidth. After removing the transient intervals originating from the switching process, we compare the dynamics induced by the short feedback loop with the corresponding dynamics induced by the replay from the long loop to test consistency of the responses of the laser. An illustration of

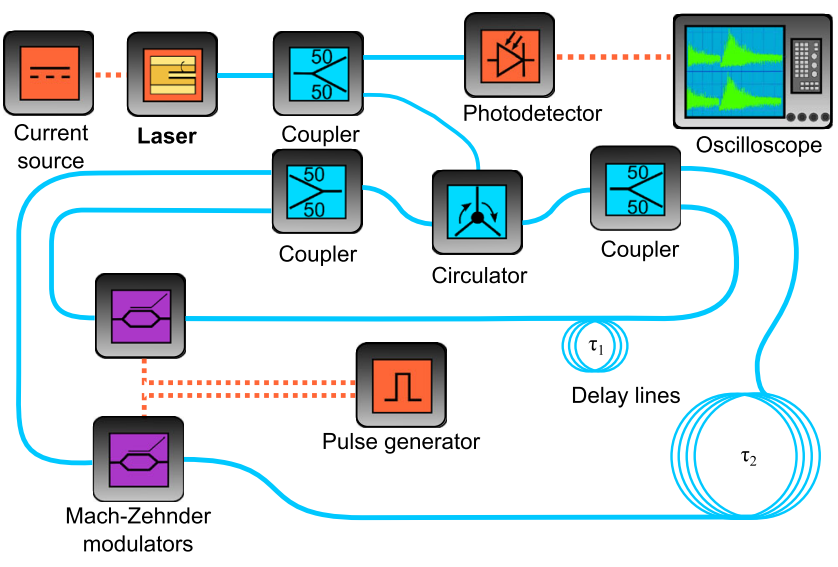

FIG. 1 (color online). Experimental consistency setup. the dynamical performance of the experiment is shown in Fig. 2.

The laser intensity output for the short loop cycle and for the long loop cycle are plotted in a spatiotemporal representation [Figs. 2(a) and 2(b), respectively] [28]. In such plots, the output power of the laser is color coded; the horizontal axis represents the time up to the round-trip time $\tau_{1}$ and the vertical axis displays the repetitions of $\tau_{1}$ within each cycle. For better visualization, only a zoom is shown [12]. The dynamics is characterized by chaotic intensity fluctuations including irregular power dropouts. The dynamics induced by the short and long feedback loop exhibit the same patterns, illustrating the high accuracy of the replay and the consistency properties for the given conditions. The major differences, displayed as dark horizontal lines in Fig. 2(b), correspond to additional noise-induced dropouts occurring in the replay. Because the drive is the same, these dropouts are not maintained after one delay time $\tau_{1}$, meaning that the system in the long loop cycle catches up to the original patterns and restores consistency. Although Fig. 2 illustrates the good correspondence between the two responses, this is not a general feature. It is rather related to the particular dynamical regime, which corresponds to a high level of consistency.

In contrast, Fig. 3 shows spatiotemporal plots of the laser output for the short (a) and long (b) loop acquired for a different pump current $\left(I=1.27 I_{\mathrm{th}}\right)$, by which we vary the dynamical properties. The laser dynamics are characterized by different chaotic fluctuations. Similar structures can be identified in the plots of both loops. However, there is not a one-to-one correspondence between Figs. 3(a) and 3(b), even though the laser received the same drive. This means that the response of the laser to its feedback is inconsistent here. Both results demonstrate that the laser can exhibit different and independent responses to the same drive depending on the dynamical regime, indicating different levels of consistency.

Consistency of the laser response is directly quantified by the cross-correlation coefficient between the power
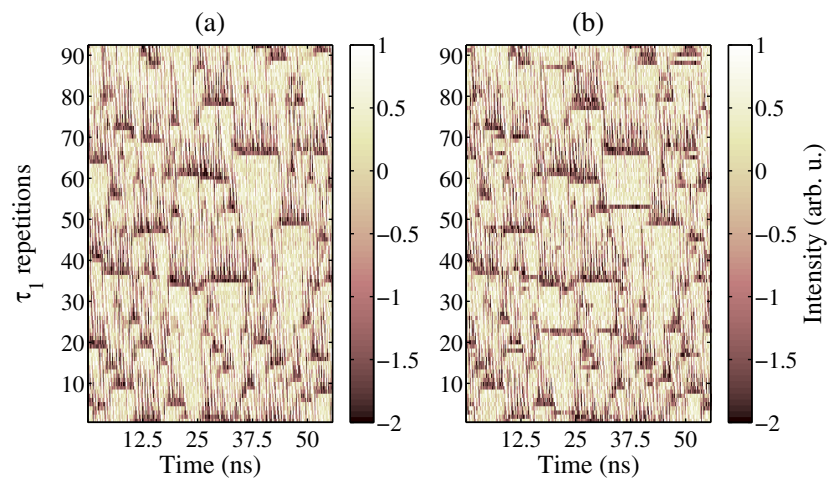

FIG. 2 (color online). Zoom of the space-time plots of the laser output acquired at $I=I_{\text {th }}$ for the (a) short loop cycle and (b) long loop cycle. 
(a)

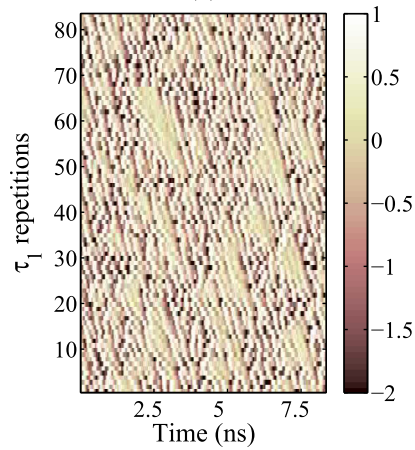

(b)

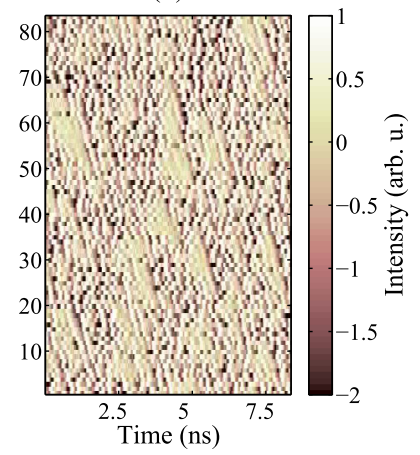

FIG. 3 (color online). Zoom of the space-time plots of the laser output acquired at $I=1.27 I_{\text {th }}$ for the (a) short loop cycle and (b) long loop cycle.

output signals $x_{1}(t)$ and $x_{2}(t)$ from the short and the long loop cycle, respectively. We denote it as the consistency correlation $C_{c}=\left\langle x_{1}(t) x_{2}(t)\right\rangle$, where each segment is normalized such that $\left\langle x_{i}(t)\right\rangle=0$ and $\left\langle x_{i}^{2}(t)\right\rangle=1, i=\{1,2\}$, and the time arguments have been deskewed by $\tau_{2}-\tau_{1}$. Additionally to this measure, we evaluate the autocorrelation function for each of the cycles and determine the maximum correlation of the first delay echo [25], which was used as an indicator for strong and weak chaos. We calculate $C_{i}=\left\langle x_{i}(t) x_{i}\left(t-t_{i}\right)\right\rangle, i=\{1,2\}$, with the same normalization as above and $t_{1} \approx t_{2} \approx \tau_{1}$ being the peak positions close to the short delay for the short and long loop cycle, respectively. Figure 4 shows these three correlation measures as a function of the pump current.

We observe that all of the selected correlation coefficients follow the same trend, developing a single minimum

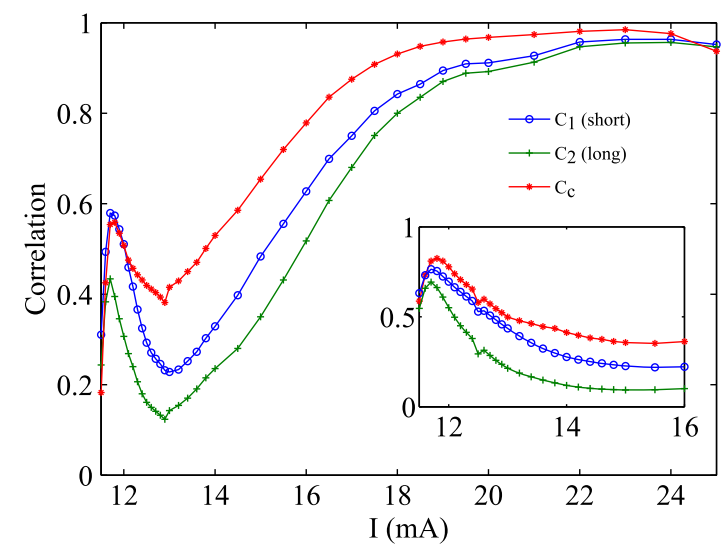

FIG. 4 (color online). Correlation coefficients as a function of the pump current. Blue and green curves show the autocorrelation coefficients for the short and long loop cycle, respectively. The red curve represents the consistency correlation. The inset shows the correlation coefficients for higher feedback strength, recorded with amplification of the detected signal. Peak positions $t_{1}$ and $t_{2}$ depend on the pump current and are located close to $\tau_{1}$ within a margin of 50 ps. at moderately low pump currents and then rising and saturating for higher pump currents. Consistency correlations up to $C_{c} \approx 0.99$ exemplify the high quality of the replay setup. The minimum of all correlation coefficients around $I=13 \mathrm{~mA}$ indicates a possible region of strong chaos interrupting weak chaos for high and low pump currents. At high pump currents the values of $C_{1}$ and $C_{2}$ converge, because the signals in cycle 1 and 2 are practically identical. Around the minimum the discrepancy between $C_{1}$ and $C_{2}$ is largest. It originates from consistency properties rather than a mismatch of the drive signals. Analyzing the entire autocorrelation functions, we find that the difference is only affecting the delay echoes, whereas the zero-peak structure is identical in both cycles [12]. The correlation coefficient $C_{1}$ measures the response of the laser to its own $\tau_{1}$-delayed signal. In contrast, $C_{2}$ measures a different relationship, because due to the inconsistency the signal $x_{2}\left(t-\tau_{1}\right)$ is not the input underlying $x_{2}(t)$, but $x_{1}\left(t-\tau_{1}\right)$, which is only structurally similar, like a surrogate. The lost information is reflected in the gap between $C_{1}$ and $C_{2}$ and vanishes with increasing consistency.

In order to study the transition from weak to strong chaos at low pump currents, we increased the feedback strength by almost a factor 2 , changing the coupler located at the laser output for a 90:10 one. A semiconductor optical amplifier is placed in the detection path to overcome the subsequent loss in the detected signal power. The resulting correlation coefficients are shown in the inset of Fig. 4. The correlation measures show a stronger increase with a maximum around $I=11.8 \mathrm{~mA}$. The consistency correlation at this point goes beyond $C_{c}>0.8$, corroborating the region of weak chaos. For increasing pump current, again the correlation functions develop a minimum. They are expected to recover to high values for even higher pump currents [25].

Subtraction of signals from the short delay cycle $x_{1}(t)$ and the long delay cycle $x_{2}(t)$ reveals the transverse signal $v(t)=x_{1}(t)-x_{2}(t)$. The term transverse refers to the corresponding generalized synchronization setup, in which $v(t)$ is the synchronization error, i.e., a projection of the phase space components transverse to the synchronization manifold. With normalization as above, the variance of the transverse signal is directly connected to the consistency correlation by $C_{c}=1-\left\langle v^{2}\right\rangle / 2$. Additional information can be obtained from the distribution function of $v(t)$. For complete synchronization of coupled discrete maps it was shown that the transverse component follows a power law distribution $p(v) \propto|v|^{\sigma-1}$ [9]. The power law exponent $\sigma$ is directly related to the transverse Lyapunov exponent $\lambda_{t}$, such that close to the synchronization threshold $\sigma \propto \lambda_{t}$. Although for continuous systems with vector-valued state variables a complete theory is not yet developed, we show how in analogy to the case of discrete maps it is possible to extract a power law exponent $\sigma$ that is proportional to the sub-LE $\lambda_{0}$. By definition, $\lambda_{0}$ results from the exponential 
evolution of the transverse coordinate $\mathbf{v}(t)=\mathbf{x}_{1}(t)-\mathbf{x}_{2}(t)$, where $\mathbf{x}_{1}, \mathbf{x}_{2}$ denote the $B$-dimensional vector state of the system, from which we can only access a scalar projection. Assuming that $\mathbf{v}(t)$ describes a small perturbation governed by linearized equations of motion, the logarithm of the perturbation size $l_{v}(t)=\ln \|\mathbf{v}(t)\|$ leads to $\lambda_{0}=\left\langle d l_{v}(t) / d t\right\rangle_{t}$ as its average growth rate. In agreement with this definition, we can write a differential equation $d l_{v}(t) / d t=\lambda_{0}+\eta(t)$ with a term $\eta(t)$ incorporating the finite time fluctuations of the Lyapunov exponent such that $\langle\eta(t)\rangle_{t}=0$. Assuming the same limiting and reinjection mechanisms as in the corresponding theory for the discrete maps, we expect an exponential distribution $\rho\left(l_{v}\right) \propto \exp \left(\sigma l_{v}\right)$ with $\sigma=\lambda_{0} / D$. The scaling factor $D$ is related to the intensity of the fluctuations in $\eta(t)$. If $\eta(t)$ were white noise, its spectral power density would be $2 D$ accordingly. The exponential distribution holds only in the regime governed by the linear equations of motion. For small arguments $l_{v}$ the distribution is dominated by noise and parameter mismatches, which in the double delay loop experiment only stem from the minimized differences between the two feedback loops. The resulting high quality of the transverse signal allows for the analysis of the distribution function $\rho\left(l_{v}\right)$. For large arguments $l_{v}$, the distribution is cut off by nonlinearities because the transverse coordinate is bounded by the typical standard deviation of the trajectories. To obtain the transverse distribution function from the experimental time traces $x_{1}(t)$ and $x_{2}(t)$, a reconstruction of the vector states $\mathbf{x}_{1}(t)$ and $\mathbf{x}_{2}(t)$ is necessary. We choose the canonical delay embedding with an embedding dimension $b$ and an embedding delay $h$, such that each vector state is approximated by $\mathbf{x}_{i}(t) \sim \mathbf{y}_{i}(t)=\left[\left(x_{i}(t), x_{i}(t-h), \ldots, x_{i}(t-(b-1) h)\right]^{\top}\right.$, $i=\{1,2\}$. Although the embedding reconstruction is not guaranteed formally by Taken's theorem, because the signals originate from externally driven systems, the reconstruction error is expected to be reduced in the transverse signal, because the explicit dependence on the external drive vanishes here. Thus, we calculate the logarithmic distance $l_{v}(t)=\ln \left\|\mathbf{y}_{1}(t)-\mathbf{y}_{2}(t)\right\|$ for different values of the embedding dimension and embedding delay.

As a complement, we have numerically simulated the corresponding scheme using the Lang-Kobayashi rate equations and applied the same analysis in order to corroborate the above assumptions. Remarkably, we found consistent results for the decay rate $\sigma$ of the distribution functions throughout all embedding dimensions and embedding delays. We further were able to confirm the proportionality $\sigma \propto \lambda_{0}$ by comparison with the directly calculated Lyapunov exponent from linearizations [12].

In the experimental distribution functions $l_{v}$, we can identify the expected three regimes. We determine the slope $\sigma$ in the useful intermediate region by using the plateau of the slope $d \ln \left(\rho\left(l_{v}\right)\right) / d l_{v}$ from the set of curves for different embedding dimensions. The results of this analysis are

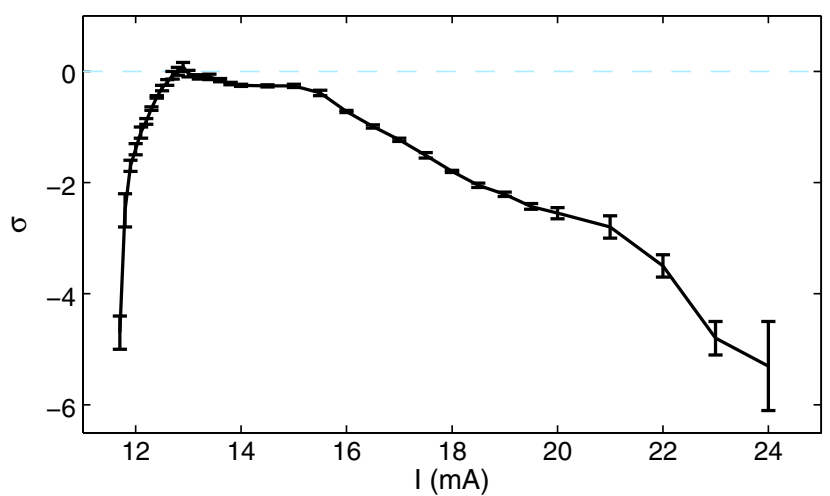

FIG. 5 (color online). Slope $\sigma=d \ln \left(\rho\left(l_{v}\right)\right) / d l_{v}$ in the intermediate regime of the transverse distribution functions. For the calculation of $l_{v}$ we set the embedding delay $h$ to the first zero crossing of the autocorrelation function from the time traces, and the embedding dimensions range from $b=2$ to $b=7$. Error bars denote the quality of convergence with increasing embedding dimension thus not containing systematic errors. For a detailed description of the method see Ref. [12].

summarized in Fig. 5, depicting the dependence of $\sigma$ on the pump current. Starting with low values of $\sigma$ for small currents, the curve develops a maximum where the correlations are minimum, and again decays for large currents. We infer a large negative value of the sub-LE in the regimes of high consistency correlation, which means that intermittent bursts of the transverse variable are comparably rare, are of low amplitude, and quickly recover towards periods of full consistency. The power law distribution of $v$ with an exponent $\sigma-1$ supports this picture, as for the negative $\sigma$ there is a pronounced peak at $v=0$ with a rapid decay. For intermediate pump currents, at the studied conditions we obtain only a slight zero crossing of $\sigma$ and thus of $\lambda_{0}$. Simulations indicate that this is related to the $\alpha$ parameter of the employed laser, being $\alpha \sim 2$ [12]. Interestingly, a marginal sub-LE already causes a very low consistency correlation. This shows the role of the finite-time fluctuations $\eta(t)$ of the sub-LE, which lead to large and frequent excursions from the synchronized state. Hence, the studies on the transverse distributions complement and extend those of the correlation functions. Recalling the direct connection between the sub-LE and $\sigma$, which was verified in simulations, we demonstrated the direct determination of the sub-LE from experimental data.

In conclusion, we presented an optical consistency experiment with nonscalar drives showing that semiconductor lasers may respond in a consistent or inconsistent way depending on feedback conditions. The different consistency regimes are directly related to the sub-LE of the system. While up to now no experimental access to the sub-LE of the system existed, we developed an experimental method to directly measure the consistency properties for high optical and dynamical bandwidth drives. By means of the analysis of transverse distribution functions 
we could extract a direct signature of the sub-LE. With the relevance and implications of consistency in various fields, we expect this method to prove valuable for further fundamental studies and applications.

T. J. and N. O. have contributed equally to the Letter. This work was supported by MINECO (Spain), Comunitat Autònoma de les Illes Balears and FEDER under Projects TEC2012-36335 (TRIPHOP), and Grups Competitius. N.O. is funded by a JAE-PreDoc grant from CSIC and cofunded by Programa Operativo FSE 2007-2013. T. J. acknowledges support by a fellowship within the Postdoc-Programme of the German Academic Exchange Service (DAAD).

*thomas@ifisc.uib-csic.es

[1] A. Uchida, R. McAllister, and R. Roy, Phys. Rev. Lett. 93, 244102 (2004).

[2] Z. Mainen and T. J. Sejnowski, Science 268, 1503 (1995).

[3] W. Maass, T. Natschläger, and H. Markram, Neural Comput. 14, 2531 (2002).

[4] L. Appeltant, M. Soriano, G. Van der Sande, J. Danckaert, S. Massar, J. Dambre, B. Schrauwen, C. Mirasso, and I. Fischer, Nat. Commun. 2, 468 (2011).

[5] L. Larger, M. C. Soriano, D. Brunner, L. Appeltant, J. M. Gutierrez, L. Pesquera, C. R. Mirasso, and I. Fischer, Opt. Express 20, 3241 (2012).

[6] Y. Paquot, F. Duport, A. Smerieri, J. Dambre, B. Schrauwen, M. Haelterman, and S. Massar, Sci. Rep. 2, 287 (2012).

[7] F. Duport, B. Schneider, A. Smerieri, M. Haelterman, and S. Massar, Opt. Express 20, 22783 (2012).

[8] D. Brunner, M. C. Soriano, C. R. Mirasso, and I. Fischer, Nat. Commun. 4, 1364 (2013).

[9] A. Pikovsky, M. Rosenblum, and J. Kurths, Synchronization: A Universal Concept in Nonlinear Sciences (Cambridge University Press, Cambridge, England, 2003), Vol. 12.

[10] N. F. Rulkov, M. M. Sushchik, L. S. Tsimring, and H. D. I. Abarbanel, Phys. Rev. E 51, 980 (1995).
[11] A. Uchida, R. McAllister, R. Meucci, and R. Roy, Phys. Rev. Lett. 91, 174101 (2003).

[12] See Supplemental Material at http://link.aps.org/ supplemental/10.1103/PhysRevLett.114.123902 for a discussion of consistency vs. generalized synchronization, details of the experimental and parameter extraction methods, as well as numerical simulations.

[13] H. D. I. Abarbanel, N. F. Rulkov, and M. M. Sushchik, Phys. Rev. E 53, 4528 (1996).

[14] L. M. Pecora and T. L. Carroll, Phys. Rev. Lett. 64, 821 (1990).

[15] T. Yamamoto, I. Oowada, H. Yip, A. Uchida, S. Yoshimori, K. Yoshimura, J. Muramatsu, S.-I. Goto, and P. Davis, Opt. Express 15, 3974 (2007).

[16] I. Oowada, H. Ariizumi, M. Li, S. Yoshimori, A. Uchida, K. Yoshimura, and P. Davis, Opt. Express 17, 10025 (2009).

[17] K. Kanno and A. Uchida, Phys. Rev. E 86, 066202 (2012).

[18] H. Aida, M. Arahata, H. Okumura, H. Koizumi, A. Uchida, K. Yoshimura, J. Muramatsu, and P. Davis, Opt. Express 20, 11813 (2012).

[19] S. Sunada, K. Arai, K. Yoshimura, and M. Adachi, Phys. Rev. Lett. 112, 204101 (2014).

[20] K. Pyragas, Phys. Rev. E 56, 5183 (1997).

[21] A. Uchida, K. Yoshimura, P. Davis, S. Yoshimori, and R. Roy, Phys. Rev. E 78, 036203 (2008).

[22] M. C. Soriano, J. García-Ojalvo, C. R. Mirasso, and I. Fischer, Rev. Mod. Phys. 85, 421 (2013).

[23] S. Heiligenthal, T. Dahms, S. Yanchuk, T. Jüngling, V. Flunkert, I. Kanter, E. Schöll, and W. Kinzel, Phys. Rev. Lett. 107, 234102 (2011).

[24] S. Heiligenthal, T. Jüngling, O. D’Huys, D. A. ArroyoAlmanza, M. C. Soriano, I. Fischer, I. Kanter, and W. Kinzel, Phys. Rev. E 88, 012902 (2013).

[25] X. Porte, M. C. Soriano, and I. Fischer, Phys. Rev. A 89, 023822 (2014).

[26] D. Arroyo-Almanza, A. Pisarchik, I. Fischer, C. Mirasso, and M. Soriano, Opt. Commun. 301-302, 67 (2013).

[27] W.-H. Kye, M. Choi, M.-S. Kurdoglyan, C.-M. Kim, and Y.-J. Park, Phys. Rev. E 70, 046211 (2004).

[28] F.-T. Arecchi, G. Giacomelli, A. Lapucci, and R. Meucci, Phys. Rev. A 45, R4225 (1992). 